PROCEEDINGS OF THE

AMERICAN MATHEMATICAL SOCIETY

Volume 141, Number 3, March 2013, Pages 883-893

S 0002-9939(2012)11365-6

Article electronically published on July 5, 2012

\title{
OPTIMAL UPPER BOUNDS FOR THE EIGENVALUE RATIOS OF ONE-DIMENSIONAL $p$-LAPLACIAN
}

\author{
CHAO-ZHONG CHEN, C. K. LAW, WEI-CHENG LIAN, AND WEI-CHUAN WANG
}

(Communicated by Yingfei Yi)

\begin{abstract}
We give optimal upper bounds for the Dirichlet and Neumann eigenvalue ratios of the one-dimensional $p$-Laplacian with nonnegative potentials. In case the potential is single-well, the upper bound for the Dirichlet eigenvalue ratios can be further refined.
\end{abstract}

\section{INTRODUCTION}

Consider the Sturm-Liouville equation with Dirichlet boundary conditions on $(0,1)$ :

$$
\begin{aligned}
-y^{\prime \prime}+q(x) y & =\lambda y \\
y(0)=y(1) & =0 .
\end{aligned}
$$

It is well known that there exists a sequence of simple isolated eigenvalues $\lambda_{n}$ such that

$$
\lambda_{1}<\lambda_{2}<\lambda_{3}<\cdots,
$$

and the associated eigenfunction $y_{n}$ has exactly $n$ simple zeros in $[0,1)$. The issues of optimal estimates for the eigenvalue ratios $\frac{\lambda_{n}}{\lambda_{m}}$ and eigenvalue gaps $\lambda_{n}-\lambda_{m}$ (where $n>m$ ) have attracted a lot of attention (cf. [13, 8, 10]). In 1989, Ashbaugh and Benguria 1 studied the optimal upper bound of eigenvalue ratios using a modified Prüfer substitution

$$
y=r(x) \sin (\sqrt{\lambda} \theta(x)), \quad y^{\prime}=r(x) \sqrt{\lambda} \cos (\sqrt{\lambda} \theta(x)),
$$

which seems to be very useful in understanding the general behavior of SturmLiouville operators.

Theorem 1.1. For the Dirichlet Sturm-Liouville problem (1.1), (1.2), if $q \geq 0$, and $n>m \geq 1$, then

$$
\frac{\lambda_{n}}{\lambda_{m}} \leq\left(\left\lceil\frac{n}{m}\right\rceil\right)^{2} .
$$

Equality holds if and only if $q=0$ and $n$ is an integral multiple of $m$.

Received by the editors June 6, 2009 and, in revised form, July 6, 2011 and July 12, 2011.

2010 Mathematics Subject Classification. Primary 34A34, 34L15.

Key words and phrases. p-Laplacian, eigenvalue ratio, single-well, generalized Prüfer substitution.

The second and third authors were partially supported by the National Science Council, Taiwan, R.O.C., under contract numbers NSC 97-2115-M-110-005-MY2 and NSC 98-2115-M-022-001. 
Later, Huang and Law [11] extended the results in [2] to more general boundary conditions, in particular, Neumann boundary conditions

$$
y^{\prime}(0)=y^{\prime}(1)=0 \text {. }
$$

Let $\mu_{n}$ be the $n$th eigenvalue for the Neumann problem, whose associated eigenfunction has exactly $n$ zeros in $[0,1)$.

Theorem 1.2. For the Neumann Sturm-Liouville problem (1.1), (1.4), if $q \geq 0$, and $n>m \geq 1$, then

$$
\frac{\mu_{n}}{\mu_{m}} \leq\left(2\left\lfloor\frac{1}{2}\left\lceil\frac{n}{m}\right\rceil\right\rfloor+1\right)^{2} .
$$

Equality holds if and only if $q=0$ and $n$ is an odd multiple of $m$.

At the end of the paper [1], Ashbaugh and Benguria posed the problem of dropping the ceiling function in Theorem 1.1 if $q$ is assumed to be convex. Recently Horváth and Kiss [9] gave a positive answer.

Theorem 1.3. For the Dirichlet Sturm-Liouville problem (1.1), (1.2), if $q \geq 0$ and is a single-well function on $(0,1)$, then for any $n>m \geq 1$,

$$
\frac{\lambda_{n}}{\lambda_{m}} \leq\left(\frac{n}{m}\right)^{2}
$$

Equality holds if and only if $q=0$ in $(0,1)$.

Here $f$ is a single-well function on $[0,1]$ if there is a point $a \in[0,1]$ such that $f$ is decreasing on $[0, a]$ and increasing on $[a, 1]$. Note that convex functions and constant functions are also single-well.

In their paper [9], Horváth and Kiss made use of another modified Prüfer substitution

and then let $\psi=\frac{\varphi}{\omega}$, so that

$$
y(x)=\frac{\rho}{\omega} \sin \varphi, \quad y^{\prime}(x)=\rho \cos \varphi
$$

$$
y(x)=\frac{\rho}{\omega} \sin \omega \psi, \quad y^{\prime}(x)=\rho \cos \omega \psi .
$$

This modified phase $\psi$ is very similar to the $\theta$ function given in (1.3). Their method is to show that as long as $q$ is decreasing on $[0, a], \varphi^{\prime}(x)>0$ and $\psi(a, \omega)$ is also a strictly increasing function of $\omega$. Then they defined

$$
\Phi(\omega)=\psi(a, w)+\tilde{\psi}(1-a, \omega),
$$

where $\tilde{\psi}$ is the modified Prüfer substitution corresponding to the potential $\tilde{q}(x)=$ $q(1-x)$. So if $q$ is single-well, then $\Phi$ is increasing in $\omega$, and at $\omega_{n}=\sqrt{\lambda_{n}}$, $\Phi\left(\omega_{n}\right)=\frac{n \pi}{\omega_{n}}$ after some computations, and hence Theorem 1.3 is valid.

It has now been understood that the $p$-Laplacian eigenvalue problem

$$
\begin{aligned}
-\nabla \cdot\left(|\nabla y|^{p-2} \nabla y\right)+q y^{(p-1)} & =\lambda y^{(p-1)}, \\
\left.y\right|_{\partial \Omega}=0, & \text { or }\left.\quad \frac{\partial y}{\partial n}\right|_{\partial \Omega}=0,
\end{aligned}
$$

where $f^{(p-1)}=|f|^{p-1} \operatorname{sgn} f=|f|^{p-2} f$ has very similar properties as the classical case when $p=2$, especially in the one-dimensional case (cf. [7, 14, 3]). In that case, the problem becomes

$$
-\left[\left(y^{\prime}\right)^{(p-1)}\right]^{\prime}=(p-1)(\lambda-q(x)) y^{(p-1)},
$$


with Dirichlet boundary conditions

$$
y(0)=y(1)=0
$$

or Neumann boundary conditions

$$
y^{\prime}(0)=y^{\prime}(1)=0 .
$$

The classical Sturm-Liouville problem corresponds to the case when $p=2$. Now a generalized Prüfer substitution helps to establish the classical Sturm-Liouville properties in that there is an increasing sequence of simple eigenvalues $\lambda_{n}\left(\mu_{n}\right)$ such that the associated eigenfunction $y_{n}$ has $n$ zeros in $[0,1)([3,14)$. Our main results are

Theorem 1.4. For the Dirichlet eigenvalue problem (1.5) with (1.6), if $q$ is nonnegative and continuous, then

$$
\frac{\lambda_{n}}{\lambda_{1}} \leq n^{p} .
$$

Moreover, equality holds if and only if $q=0$.

Theorem 1.5. For the Neumann eigenvalue problem (1.5) with (1.7), if $q$ is nonnegative and continuous, then

$$
\frac{\mu_{n}}{\mu_{1}} \leq\left(2\left\lfloor\frac{n}{2}\right\rfloor+1\right)^{p}
$$

Equality holds if and only if $q=0$ and $n$ is odd.

Theorem 1.3 can also be extended to work for the one-dimensional $p$-Laplacian.

Theorem 1.6. For the one-dimensional p-Laplacian (1.5) with Dirichlet boundary condition (1.6), if $q \geq 0$ is a single-well function, then

$$
\frac{\lambda_{n}}{\lambda_{m}} \leq\left(\frac{n}{m}\right)^{p} .
$$

Equality holds if and only if $q=0$ in $(0,1)$.

We find that the method of Horváth-Kiss cannot be extended directly. The main difficulty is in the proof for the analogue of $\varphi^{\prime}>0$. Since $\theta$ as given in (1.3) is a commonly used modified Prüfer substitution in studying the eigenvalue ratio, we try the associated generalized Prüfer substitution instead of the analogues of $\phi$ and $\psi$ and the proof goes through.

Furthermore we also have an extension of [9, Corollary 2.3] for Dirichlet-Neumann boundary conditions, whence

$$
\frac{\lambda_{n}}{\lambda_{m}} \leq \frac{(2 n-1)^{p}}{(2 m-1)^{p}}
$$

The proof is standard and will be omitted.

In section 2, we shall introduce a generalized Prüfer substitution and develop some of its properties. Then we shall prove Theorem 1.4 and Theorem 1.5 in section 3. In section 4, we shall prove Theorem 1.6 .

Finally we remark that Theorems $1.1,1.3$ are now special cases of our theorems. It seems that the one-dimensional $p$-Laplacian inherits most of the Sturm-Liouville properties that are related to Prüfer substitution. Our results add more evidence to the above statement. 


\section{Preliminaries}

Fix $p>1$. When $q=0$, equation (1.5) becomes

$$
-\left[\left(y^{\prime}\right)^{(p-1)}\right]^{\prime}=(p-1) \lambda y^{(p-1)} .
$$

If $\lambda=1$, let $S$ be the solution of this equation satisfying the initial conditions $S_{p}(0)=0, \quad S_{p}^{\prime}(0)=1$. The functions $S$ and $S^{\prime}$ are periodic functions on $\mathbf{R}$. They are in fact $p$-analogues of sine and cosine functions in the classical case. It is well known that $\widehat{\pi}=\frac{2 \pi}{p \sin \left(\frac{\pi}{p}\right)}$ is the first zero of $S$. The following lemma was proved by Elbert [7].

Lemma 2.1 ([7]). (a) $\quad\left|S_{p}(x)\right|^{p}+\left|S_{p}^{\prime}(x)\right|^{p}=1$ for any $x \in \mathbf{R}$.

(b) $\quad\left|S_{p}^{\prime}(x)\right|^{p-2} S_{p}^{\prime \prime}(x)=-S_{p}^{(p-1)}(x)$ for any $x \in \mathbf{R}$.

Let $T_{p}(x)=\frac{S_{p}(x)}{S_{p}^{\prime}(x)}$. Then one can easily derive $T_{p}^{\prime}(x)=1+\left|T_{p}(x)\right|^{p}$ for any $x \in \mathbf{R}$. Also,

Lemma 2.2. If $0<|t|<\frac{\widehat{\pi}}{2}$, then $T_{p}(t)>t$.

Proof. Let $g(t)=T_{p}(t)-t$. Then $g^{\prime}(t)=T_{p}^{\prime}(t)-1>0$. Thus $g^{\prime}>0$, and $T_{p}(t)>t$.

We consider a generalized Prüfer substitution on the one-dimensional $p$-Laplacian (1.5). With $\rho:=\lambda^{1 / p}$, we have

$$
y(x)=R(x) S_{p}(\rho \Theta(x)), \quad y^{\prime}(x)=R(x) \rho S_{p}^{\prime}(\rho \Theta(x)),
$$

so that

$$
\frac{y^{\prime}}{y}=\frac{\rho S_{p}^{\prime}(\rho \Theta(x))}{S_{p}(\rho \Theta(x))} .
$$

Differentiate both sides with respect to $x$. Then from Lemma 2.1(b) and (1.5), with $S_{p}^{\prime}$ being the derivative of $S_{p}$ with respect to its argument, we have part (a) of the following lemma.

Lemma 2.3. Let $\rho=\lambda^{1 / p}$.

$$
\begin{aligned}
& \text { (a) } \Theta^{\prime}(x)=1-\frac{q(x)}{\rho^{p}}\left|S_{p}(\rho \Theta(x))\right|^{p}, \\
& \text { (b) } R^{\prime}(x)=\rho^{1-p} q(x) R(x) S_{p}(\rho \Theta(x))^{(p-1)} S_{p}^{\prime}(\rho \Theta(x)) .
\end{aligned}
$$

Proof. (b) From (2.1), we have

$$
R(x)^{p}=y(x)^{p}+\left(\frac{y^{\prime}(x)}{\rho}\right)^{p} .
$$

Differentiate (2.4) and simplify to yield

$$
\begin{aligned}
R^{\prime} & =R \rho S_{p}^{(p-1)} S_{p}^{\prime}-\frac{\left(\rho^{p}-q\right) R S_{p}^{\prime} S_{p}^{(p-1)}}{\rho^{p-1}} \\
& =q \rho^{1-p} R S_{p}^{(p-1)} S_{p}^{\prime} .
\end{aligned}
$$

Therefore, we get (2.3). 
Lemma 2.4. Suppose $c \geq 1,|x| \leq \frac{\lfloor c\rfloor \widehat{\pi}}{c}$. Then

$$
c^{p}\left|S_{p}(x)\right|^{p} \geq\left|S_{p}(c x)\right|^{p} .
$$

Proof. Let

$$
f(x)=c S_{p}(x) \pm S_{p}(c x), 0 \leq x \leq \frac{\lfloor c\rfloor \widehat{\pi}}{c} .
$$

For any critical value $\Theta_{c}$, we have $f^{\prime}\left(\Theta_{c}\right)=0$. That is, $S_{p}^{\prime}\left(\Theta_{c}\right)= \pm S_{p}^{\prime}\left(c \Theta_{c}\right)$, so that $S_{p}\left(\Theta_{c}\right)= \pm S_{p}\left(c \Theta_{c}\right)$, which in turn implies $f\left(\Theta_{c}\right)=(c \pm 1) S_{p}\left(\Theta_{c}\right) \geq 0$. Furthermore, $f(0)=0$ and $f\left(\frac{\lfloor c\rfloor \widehat{\pi}}{c}\right)=c S_{p}\left(\frac{\lfloor c\rfloor \widehat{\pi}}{c}\right) \geq 0$. So we conclude that for all $0 \leq x \leq \frac{\lfloor c\rfloor \widehat{\pi}}{c}, c S_{p}(x) \pm S_{p}(c x) \geq 0$. Therefore $c^{p}\left|S_{p}(x)\right|^{p} \geq\left|S_{p}(c x)\right|^{p}$.

\section{Nonnegative potentials}

Let $y_{j}$ denote the normalized eigenfunctions corresponding to the eigenvalue $\lambda_{j}$ $(j \in \mathbf{N})$. Then to each $y_{j}$ we can associate a Prüfer angle via the transformation (2.1). Letting $\Theta_{j}(0)=0$ for $j=1,2,3, \ldots$, one has

$$
\Theta_{j}(1)=j \widehat{\pi} / \sqrt[p]{\lambda_{j}} \text { for } j=1,2,3, \ldots
$$

Lemma 3.1. Suppose that $q$ is nonnegative. Then all the Dirichlet and Neumann eigenvalues of the one-dimensional p-Laplacian (1.5), except for the first Neumann eigenvalue, are positive.

Proof. We use the classical generalized Prüfer substitution: $y=r(x) S_{p}(\Theta(x)), y^{\prime}=$ $r(x) S_{p}^{\prime}(\Theta(x))$. Then

$$
\Theta^{\prime}(x)=(\lambda-q)\left|S_{p}(\Theta(x))\right|^{p}+\left|S_{p}^{\prime}(\Theta(x))\right|^{p} .
$$

Note that the above equation can be found in 3 . Then when $q \geq 0$ and $\lambda \leq 0$, we have

$$
\Theta^{\prime}(x) \leq\left|S_{p}^{\prime}(\Theta(x))\right|^{p}
$$

Consider the equation $\left(\bar{y}^{\prime(p-1)}\right)^{\prime}=0$. It is easy to see that the general solution is exactly $\bar{y}=C_{1} x+C_{2}$, for constants $C_{1}, C_{2}$. Observe that the associated phase $\bar{\Theta}(x)$ for this equation is $\bar{\Theta}^{\prime}(x)=\left|S_{p}^{\prime}(\bar{\Theta}(x))\right|^{p}$. Hence by a comparison theorem ([11, p. 1431]; see also [4, p. 30]), $\Theta(x) \leq \bar{\Theta}(x)$ for all $x \in(0,1]$. Now for Dirichlet boundary conditions, $C_{2}=0$ and $C_{1} \geq 0$. Hence $\bar{y}>0$ which means that $\bar{\Theta}$ and so $\Theta$ never reaches $\widehat{\pi}$. For Neumann boundary conditions, $C_{1}=0$ and $y=C_{2} \neq 0$. So $\bar{\Theta} \equiv \widehat{\pi} / 2$, and the same conclusion holds.

Proof of Theorem 1.4. Suppose that (1.8) is not true, i.e.,

$$
\frac{\lambda_{n}}{\lambda_{1}}>n^{p} \text {. }
$$

Then it follows from (2.2) that

$$
\begin{aligned}
\Theta_{1}^{\prime} & =1-\frac{q}{\lambda_{1}}\left|S_{p}\left(\rho_{1} \Theta_{1}\right)\right|^{p}:=F_{1}\left(x, \Theta_{1}\right), \\
\Theta_{n}^{\prime} & =1-\frac{q}{\lambda_{n}}\left|S_{p}\left(\rho_{n} \Theta_{n}\right)\right|^{p}:=F_{n}\left(x, \Theta_{n}\right) .
\end{aligned}
$$

Let $c:=\frac{\rho_{n}}{\rho_{1}}>n$ by (3.2). Also let $\varphi=\rho \Theta$. By Lemma 2.4, as long as $|\rho \Theta(x)|=$ $|c \varphi| \leq\lfloor c\rfloor \widehat{\pi}$,

$$
c^{p}\left|S_{p}(\varphi)\right|^{p} \geq\left|S_{p}(c \varphi)\right|^{p} .
$$


That is, as long as $\Theta(x)<\frac{\lfloor c\rfloor \widehat{\pi}}{\rho_{n}}$,

$$
\frac{1}{\lambda_{1}}\left|S_{p}\left(\rho_{1} \Theta\right)\right|^{p} \geq \frac{1}{\lambda_{n}}\left|S_{p}\left(\rho_{n} \Theta\right)\right|^{p} .
$$

Thus,

$$
F_{1}(x, \Theta)=1-\frac{q}{\lambda_{1}}\left|S_{p}\left(\rho_{1} \Theta\right)\right|^{p} \leq 1-\frac{q}{\lambda_{n}}\left|S_{p}\left(\rho_{n} \Theta\right)\right|^{p}=F_{n}(x, \Theta) .
$$

It follows from the comparison theorem ([11, p. 1431]; see also [4, p. 30]) that

$$
\frac{\widehat{\pi}}{\rho_{1}}=\Theta_{1}(1) \leq \Theta_{n}(1)=\frac{n \widehat{\pi}}{\rho_{n}}
$$

which implies that $\frac{\lambda_{n}}{\lambda_{1}} \leq n^{p}$. This contradicts our assumption (3.2).

Obviously when $q=0, \lambda_{1}=\widehat{\pi}^{p}$ and $\lambda_{n}=(n \widehat{\pi})^{p}$. Thus equality holds. Conversely, if $\frac{\lambda_{n}}{\lambda_{1}}=n^{p}$, then by (3.5), $\Theta_{1}(1)=\Theta_{n}(1)$. Hence by the comparison theorem, $\Theta_{1}(x)=\Theta_{n}(x)$ for all $x \in[0,1]$. That means $F_{1}\left(x, \Theta_{1}(x)\right)=F_{n}\left(x, \Theta_{n}(x)\right)$ for all $x \in[0,1]$, which is impossible unless $q=0$.

Proof of Theorem 1.5. We let

$$
\begin{array}{rlrl}
\Theta_{1}(0) & =\frac{-\widehat{\pi} / 2}{\mu_{1}^{1 / p}}, & \Theta_{n}(0) & =\frac{-\left(\left\lfloor\frac{n-1}{2}\right\rfloor+\frac{1}{2}\right) \widehat{\pi}}{\mu_{n}^{1 / p}}, \\
\Theta_{1}(1)=\frac{\widehat{\pi} / 2}{\mu_{1}^{1 / p}}, & \Theta_{n}(1)=\frac{\left(\left\lfloor\frac{n}{2}\right\rfloor+\frac{1}{2}\right) \widehat{\pi}}{\mu_{n}^{1 / p}} .
\end{array}
$$

Hence if (1.9) is not valid, then

$$
\frac{\mu_{n}}{\mu_{1}}>\left(2\left\lfloor\frac{n}{2}\right\rfloor+1\right)^{p} \geq\left(2\left\lfloor\frac{n-1}{2}\right\rfloor+1\right)^{p},
$$

which implies that $\Theta_{1}(0)<\Theta_{n}(0)$. Let $c=\left(\frac{\mu_{n}}{\mu_{1}}\right)^{1 / p}$. As above, we have $F_{1}(x, \Theta) \leq$ $F_{n}(x, \Theta)$, so that $\Theta_{1}(x) \leq \Theta_{n}(x)$ as long as $\mu_{n}^{1 / p} \Theta_{n} \leq\left(2\left\lfloor\frac{n}{2}\right\rfloor+1\right) \leq\lfloor c\rfloor \widehat{\pi}$. However, this means that

$$
\frac{\mu_{n}}{\mu_{1}}>\left(2\left\lfloor\frac{n+1}{2}\right\rfloor+1\right)^{p},
$$

which leads to a contradiction. By the comparison theorem again, equality implies that $q=0$, and $\left\lfloor\frac{n-1}{2}\right\rfloor=\left\lfloor\frac{n}{2}\right\rfloor$, which implies that $n$ is odd. Conversely, when $q=0$ and $n$ is odd, then $\mu_{1}=\widehat{\pi}^{p}$ and $\mu_{n}=(n \widehat{\pi})^{p}$.

\section{Nonnegative Single-Well potentials}

Lemma 4.1. Assume that $\Theta(0)=0$.

(a) If $q(x)>0$ is decreasing in $\left[0, x_{0}\right], \Theta^{\prime}>0$ in $\left(0, x_{0}\right)$.

(b) Let " $\dot{\Theta}$ " denote the derivative of $\Theta$ with respect to $\rho$. We have

$$
\dot{\Theta}=\frac{p}{R^{p}(x) \rho^{p+1}} \int_{0}^{x} R^{p}(t) q(t)\left(\left|S_{p}(\rho \Theta(t))\right|^{p}-\rho \Theta(t) S_{p}(\rho \Theta(t))^{(p-1)} S_{p}^{\prime}(\rho \Theta(t))\right) d t .
$$

Proof. (a) In view of Lemma 2.3 and the fact that $q$ is monotonically decreasing, it suffices to show that $\Theta^{\prime}>0$ for $0<\Theta<\min \left(x_{0}, \frac{\widehat{\pi}}{2 \rho}\right)$. First, as $\Theta(0)=0, \Theta^{\prime}(0)=1$. 
So initially $\Theta^{\prime}(x)>0$. Thus if $\Theta^{\prime}(x)>0$ for $x \in\left(0, x_{2}\right)$ for some $x_{2} \in\left(0, x_{0}\right)$. Then for all $x \in\left(0, x_{2}\right)$,

$$
1-\frac{q(x)}{\rho^{p}}\left|S_{p}(\rho \Theta(x))\right|^{p}>0,
$$

or $\rho>q(x)^{\frac{1}{p}}\left|S_{p}(\rho \Theta(x))\right|$. We assert that $\rho>q\left(x_{2}\right)^{\frac{1}{p}}\left|S_{p}\left(\rho \Theta\left(x_{2}\right)\right)\right|$ so that $\Theta^{\prime}\left(x_{2}\right)>0$. Indeed, choose $x_{1}$ arbitrarily from $\left(0, x_{2}\right)$. Now we compute

$$
\begin{aligned}
& {\left[\log \left(\rho-q(x)^{\frac{1}{p}}\left|S_{p}(\rho \Theta(x))\right|\right)\right]_{0}^{x_{1}}} \\
& \quad=\int_{0}^{x_{1}} \frac{d\left[\rho-q(x)^{\frac{1}{p}}\left|S_{p}(\rho \Theta(x))\right|\right]}{\rho-q(x)^{\frac{1}{p}}\left|S_{p}(\rho \Theta(x))\right|} \\
& \quad=\int_{0}^{x_{1}} \frac{-\left(\frac{1}{p} q^{\frac{1}{p}-1}\left|S_{p}(\rho \Theta(x))\right| d q+\rho q^{\frac{1}{p}} \Theta^{\prime} S_{p}^{\prime}(\rho \Theta) \operatorname{sgn}\left(S_{p}(\rho \Theta)\right) d x\right)}{\rho-q^{\frac{1}{p}}(x)\left|S_{p}(\rho \Theta(x))\right|} \\
& \geq-\int_{0}^{x_{1}} \frac{\rho q^{\frac{1}{p}}\left(\rho^{p}-q(x)\left|S_{p}(\rho \Theta)\right|^{p}\right)}{\rho^{p}\left(\rho-q^{\frac{1}{p}}\left|S_{p}(\rho \Theta(x))\right|\right)} d x \\
& =\int_{0}^{x_{1}}(-p) t^{p-1} \rho^{1-p} q^{\frac{1}{p}} d x
\end{aligned}
$$

where $d q \leq 0$ and $t$ lies between $\rho$ and $q(x)^{\frac{1}{p}} S_{p}(\rho \Theta(x))$ by the mean value theorem.

Now the integrand $(-p) t^{p-1}\left(\rho^{1-p} q^{\frac{1}{p}}\right)$ is bounded from below on $\left(0, x_{1}\right)$. Hence

$$
\left[\log \left(\rho-q(x)^{\frac{1}{p}}\left|S_{p}(\rho \Theta(x))\right|\right)\right]_{0}^{x_{1}} \geq-M
$$

with $M$ independent of $x_{1}$. If we let $x_{1}$ approach $x_{2}$, then $\rho>q\left(x_{2}\right)^{\frac{1}{p}}\left|S_{p}\left(\rho \Theta\left(x_{2}\right)\right)\right|$. That is, $\Theta^{\prime}\left(x_{2}\right)>0$.

(b) Differentiate (2.2) with respect to $\rho$ to obtain

$$
\dot{\Theta}^{\prime}+\frac{p q}{\rho^{p-1}} S_{p}(\rho \Theta)^{(p-1)} S_{p}^{\prime}(\rho \Theta) \dot{\Theta}=\frac{p q}{\rho^{p+1}}\left|S_{p}(\rho \Theta)\right|^{p}-\frac{p q}{\rho^{p}} S_{p}(\rho \Theta)^{(p-1)} S_{p}^{\prime}(\rho \Theta) \Theta .
$$

In view of (2.3), (4.2) becomes

$$
\left(R^{p} \dot{\Theta}\right)^{\prime}=R^{p}\left(\frac{p q}{\rho^{p+1}}\left|S_{p}(\rho \Theta)\right|^{p}-\frac{p q}{\rho^{p}} \Theta S_{p}(\rho \Theta)^{(p-1)} S_{p}^{\prime}(\rho \Theta)\right) .
$$

Thus (4.1) is valid.

Theorem 4.2. Let $q(x)$ be negative and decreasing in $\left[0, x_{0}\right]$. Then $\dot{\Theta}(x, \rho) \geq 0$. If there is a $\rho>0$ with $\dot{\Theta}(x, \rho)=0$, then $q=0$ in $\left(0, x_{0}\right]$.

We need to have one lemma before proving Theorem 4.2. From (2.3), we have

$$
\begin{array}{lll}
R^{\prime} \geq 0 & \text { when } & k \widehat{\pi} \leq \rho \Theta \leq\left(k+\frac{1}{2}\right) \widehat{\pi}, \\
R^{\prime} \leq 0 & \text { when } & \left(k+\frac{1}{2}\right) \widehat{\pi} \leq \rho \Theta \leq(k+1) \widehat{\pi},
\end{array}
$$

where $k \geq 0$ is an integer. Let $\varphi(x)=\rho \Theta(x)$. In view of Lemma 4.1)(a), $\varphi$ is strictly increasing in $x$. Denote $x_{k+r}=\varphi^{-1}((k+r) \widehat{\pi})$ for any $r \in \mathbf{R}$.

Lemma 4.3. For fixed $\rho \in \mathbb{R}, R$ is increasing on $\left(x_{k}, x_{k+\frac{1}{2}}\right)$, but decreasing on $\left(x_{k+\frac{1}{2}}, x_{k+1}\right)$. Furthermore for all $x_{0} \in\left(x_{k+1}, x_{k+\frac{3}{2}}\right), R\left(x_{0}\right) \leq R\left(x_{k+\frac{1}{2}}\right)$. 
Proof. The first part is now obvious. To prove the second part, we observe that, from (2.3), for all $x_{0} \in\left(x_{k+1}, x_{k+\frac{3}{2}}\right)$,

$$
\begin{aligned}
{[\log R(x)]_{x_{k+\frac{1}{2}}}^{x_{0}} } & =\int_{x_{k+\frac{1}{2}}}^{x_{0}} \rho^{1-p} q\left|S_{p}(\rho \Theta)\right|^{p-2} S_{p}(\rho \Theta) S_{p}^{\prime}(\rho \Theta) d x \\
& =\int_{\left(k+\frac{1}{2}\right) \widehat{\pi}}^{\varphi_{0}} \frac{\left(q \circ \varphi^{-1}\right)\left|S_{p}(\varphi)\right|^{p-2} S_{p}(\varphi) S_{p}^{\prime}(\varphi)}{\rho^{p}-\left(q \circ \varphi^{-1}\right)\left|S_{p}(\varphi)\right|^{p}} d \varphi
\end{aligned}
$$

letting $\varphi(x)=\rho \Theta(x)$. Note that $\varphi_{0} \in\left((k+1) \widehat{\pi},\left(k+\frac{3}{2}\right) \widehat{\pi}\right)$. Also since $q$ is decreasing and $S_{p}(\varphi) S_{p}^{\prime}(\varphi)$ is negative on $\left(\left(k+\frac{1}{2}\right) \widehat{\pi},(k+1) \widehat{\pi}\right)$ but positive on $\left((k+1) \widehat{\pi},\left(k+\frac{3}{2}\right) \widehat{\pi}\right)$,

$$
\begin{aligned}
\log R( & \left.x_{0}\right)-\log R\left(x_{k+\frac{1}{2}}\right) \\
\leq & \int_{\left(k+\frac{1}{2}\right) \widehat{\pi}}^{(k+1) \widehat{\pi}} \frac{q\left(x_{k+1}\right) S_{p}(\varphi)^{(p-1)} S_{p}^{\prime}(\varphi)}{\rho^{p}-q\left(x_{k+1}\right)\left|S_{p}(\varphi)\right|^{p}} d \varphi \\
& +\int_{(k+1) \widehat{\pi}}^{\varphi_{0}} \frac{\left.q\left(x_{k+1}\right)\right) S_{p}(\varphi)^{(p-1)} S_{p}^{\prime}(\varphi)}{\rho^{p}-q\left(x_{k+1}\right)\left|S_{p}(\varphi)\right|^{p}} d \varphi \\
= & \int_{\left(k+\frac{1}{2}\right) \widehat{\pi}}^{\varphi_{0}} \frac{\left.q\left(x_{k+1}\right)\right) S_{p}(\varphi)^{(p-1)} S_{p}^{\prime}(\varphi)}{\rho^{p}-q\left(x_{k+1}\right)\left|S_{p}(\varphi)\right|^{p}} d \varphi \\
= & -\frac{1}{p} \log \left|\frac{\rho^{p}-q\left(x_{k+1}\right)\left|S_{p}\left(\varphi_{0}\right)\right|^{p}}{\rho^{p}-q\left(x_{k+1}\right)}\right| \leq 0 .
\end{aligned}
$$

So $R\left(x_{0}\right) \leq R\left(x_{k+\frac{1}{2}}\right)$.

Proof of Theorem 4.2. First we note that $\dot{\Theta}(0, \rho)=0$. So as long as $\left(R^{p} \dot{\Theta}\right)^{\prime}>0$, $\dot{\Theta}>0$. Now from (4.1) and (2.3),

$$
\left(R^{p} \dot{\Theta}\right)^{\prime}=\frac{p R^{p-1} R^{\prime}}{\rho^{2}}\left(T_{p}(\rho \Theta)-\rho \Theta\right) .
$$

By the sign analyses in (4.3) and (4.4), we do have $\left(R^{p} \dot{\Theta}\right)^{\prime} \geq 0$ for $0<\rho \Theta<\frac{\widehat{\pi}}{2}$. Let $\rho \Theta\left(x_{\frac{1}{2}}\right)=\frac{\widehat{\pi}}{2}$. We have $\dot{\Theta}>0$ on $\left(0, x_{\frac{1}{2}}\right)$. In general, for $x \in\left(x_{k}, x_{k+\frac{1}{2}}\right]$, we let $\rho \Theta(x)=k \widehat{\pi}+\varphi_{k}(x)$ where $\varphi_{k}(x) \in\left(0, \frac{\widehat{\pi}}{2}\right)$. Then (4.5) yields

$$
\begin{aligned}
\left(R^{p} \dot{\Theta}\right)^{\prime} & =\frac{p R^{p-1} R^{\prime}}{\rho^{2}}\left[T_{p}\left(k \widehat{\pi}+\varphi_{k}\right)-k \widehat{\pi}-\varphi_{k}\right] \\
& =\frac{p R^{p-1} R^{\prime}}{\rho^{2}}\left[\left(T_{p}(\varphi)-\varphi\right)-k \widehat{\pi}\right] \\
& \geq-\frac{p k \widehat{\pi}}{\rho^{2}} R^{p-1} R^{\prime} .
\end{aligned}
$$


On the other hand, for $x \in\left(x_{k+\frac{1}{2}}, x_{k+1}\right]$, then $R^{\prime}<0$. Let $\rho \Theta(x)=(k+1) \widehat{\pi}-$ $\varphi_{k^{\prime}}(x)$, where $\varphi_{k^{\prime}}(x) \in\left(0, \frac{\widehat{\pi}}{2}\right)$. Thus

$$
\begin{aligned}
\left(R^{p} \dot{\Theta}\right)^{\prime} & =\frac{p R^{p-1} R^{\prime}}{\rho^{2}}\left[\left(\varphi_{k^{\prime}}-T_{p}\left(\varphi_{k^{\prime}}\right)\right)-(k+1) \widehat{\pi}\right] \\
& \geq-\frac{p(k+1) \widehat{\pi}}{\rho^{2}} R^{p-1} R^{\prime} .
\end{aligned}
$$

Hence for any $x \in\left(x_{k+\frac{1}{2}}, x_{k+\frac{3}{2}}\right)$, by Lemma 4.3 above, $\left[R^{p} \dot{\Theta}\right]_{x_{k+\frac{1}{2}}}^{x} \geq 0$. Inductively, we have $\dot{\Theta} \geq 0$.

When equality holds, then

$$
R^{\prime}(x)=R(x) \rho^{1-p} q(x) S_{p}(\rho \Theta(x))^{(p-1)} S_{p}^{\prime}(\rho \Theta(x)) \equiv 0,
$$

so that $q(x)=0$ in $\left(0, x_{0}\right]$.

Let the potential $q$ be monotone decreasing in $[0, a]$ and monotone increasing in $[a, 1]$. Denote by $\tilde{q}(x)$ the reverse of the potential, i.e., $\tilde{q}(x)=q(1-x)$. Then $\tilde{y}\left(x, \rho_{n}\right)$ is the solution of the initial value problem

$$
\left\{\begin{array}{l}
-\left[\left(\tilde{y}^{\prime}\right)^{(p-1)}\right]^{\prime}=(p-1)\left(\rho_{n}^{p}-\tilde{q}(x)\right) \tilde{y}^{(p-1)} \\
\tilde{y}(0)=0, \tilde{y}^{\prime}(0)=1
\end{array}\right.
$$

of which the modified Prüfer substitution is

$$
\left\{\begin{array}{l}
\tilde{y}\left(x, \rho_{n}\right)=\tilde{R}(x) S_{p}\left(\rho_{n} \tilde{\Theta}(x)\right) \\
\tilde{y}^{\prime}\left(x, \rho_{n}\right)=\tilde{R}(x) \rho_{n} S_{p}^{\prime}\left(\rho_{n} \tilde{\Theta}(x)\right)
\end{array}\right.
$$

and $\tilde{\Theta}(0)=0$.

Lemma 4.4. Let $y\left(1-x, \rho_{n}\right)$ be an eigenfunction for the potential $\tilde{q}$. Then

$$
\left\{\begin{array}{l}
\tilde{y}\left(x, \rho_{n}\right)=(-1)^{n+1} \frac{y\left(1-x, \rho_{n}\right)}{\rho_{n} R(1)} \\
\tilde{R}\left(x, \rho_{n}\right)=\frac{R\left(1-x, \rho_{n}\right)}{\rho_{n} R(1)} \\
\tilde{\Theta}\left(x, \rho_{n}\right)=\frac{n \pi}{\rho_{n}}-\Theta\left(1-x, \rho_{n}\right),
\end{array}\right.
$$

which satisfies $\tilde{y}\left(0, \rho_{n}\right)=0$ and $\tilde{y}^{\prime}\left(0, \rho_{n}\right)=1$.

Proof. Let $\tilde{y}(x)=c_{1} y(1-x)$. Then $\tilde{y}^{\prime}(x)=-c_{1} y^{\prime}(1-x)$ and $\tilde{y}^{\prime}\left(0, \rho_{n}\right)=1$. So $c_{1}=\frac{(-1)^{n+1}}{R(1) \rho_{n}}$. Hence

$$
\tilde{y}\left(x, \rho_{n}\right)=(-1)^{n+1} \frac{y\left(1-x, \rho_{n}\right)}{\rho_{n} R\left(1, \rho_{n}\right)} .
$$

Let $\tilde{R}(x)=c_{2} R(1-x)$. From (4.7) and the fact that $\tilde{y}^{\prime}\left(0, \rho_{n}\right)=1$, we have $c_{2}=\frac{1}{R(1) \rho_{n}}$. Hence

$$
\tilde{R}\left(x, \rho_{n}\right)=\frac{R\left(1-x, \rho_{n}\right)}{\rho_{n} R(1)} .
$$


Proof of Theorem 1.6. Consider the function $\Psi\left(\rho_{n}\right)=\Theta\left(a, \rho_{n}\right)+\tilde{\Theta}\left(1-a, \rho_{n}\right)$. By Theorem 4.2. $\Psi\left(\rho_{n}\right)$ is the sum of two monotone increasing functions and is also increasing. By (4.8), $\rho_{n} \Psi\left(\rho_{n}\right)=n \widehat{\pi}$. Let $m<n$. Then

$$
\frac{m \widehat{\pi}}{\rho_{m}}=\Psi\left(\rho_{m}\right) \leq \Psi\left(\rho_{n}\right)=\frac{n \widehat{\pi}}{\rho_{n}} .
$$

Thus $\frac{\rho_{n}}{\rho_{m}} \leq \frac{n}{m}$, which implies $\frac{\lambda_{n}}{\lambda_{m}} \leq \frac{n^{p}}{m^{p}}$.

If equality holds, then $\Psi\left(\rho_{m}\right)=\Psi\left(\rho_{n}\right)$, and hence $\Theta\left(a, \rho_{m}\right)=\Theta\left(a, \rho_{n}\right)$ and $\tilde{\Theta}\left(1-a, \rho_{m}\right)=\tilde{\Theta}\left(1-a, \rho_{n}\right)$. By Theorem 4.2 , this implies that $q=0$ in $(0, a]$ and $\tilde{q}=0$ in $(0,1-a]$, i.e., $q=0$ in $(0,1)$.

\section{FURTHER DISCUSSIONS}

1. After the first submission of our manuscript, Theorem 1.6 has been independently proved for differentiable potentials in Bognár and Dosly [5]. We thank the anonymous referee for informing us about it. We do not need the smoothness condition in our proof, and our modified Prüfer substitution is simpler.

2. Theorem 1.4 and Theorem 1.5 hold for continuous potentials. It is possible to extend the results to hold for $L^{1}$-potentials. We shall pursue the issue in another paper. On the other hand, by a similar partition argument as in [1, Prop. 3.2], one can easily show that for any $n>m \geq 1$,

$$
\frac{\lambda_{n}}{\lambda_{m}} \leq\left(\left\lceil\frac{n}{m}\right\rceil\right)^{p}, \quad \frac{\mu_{n}}{\mu_{m}} \leq\left(2\left\lfloor\frac{1}{2}\left\lceil\frac{n}{m}\right\rceil\right\rfloor+1\right)^{p} .
$$

\section{REFERENCES}

[1] M.S. Ashbaugh and R.D. Benguria, Optimal bounds for ratios of eigenvalues of onedimensional Schrödinger operators with Dirichlet boundary conditions and positive potentials, Comm. Math. Phys., 124 (1989), 403-415. MR.1012632 (91c:34114)

[2] M.S. Ashbaugh and R.D. Benguria, Eigenvalue ratios for Sturm-Liouville operators, J. Diff. Eqns., 103 (1993), 205-219. MR1218744 (94c:34125)

[3] P. Binding and P. Drabek, Sturm-Liouville theory for the p-Laplacian, Studia Scientiarum Mathematicarum Hungarica, 40 (2003), 373-396. MR2037324(2004j:34068)

[4] G. Birkhoff and G.C. Rota, Ordinary Differential Equations, 4th ed., Wiley, New York, 1989. MR.972977 (90h:34001)

[5] G. Bognár and O. Dosly, The ratio of eigenvalues of the Dirichlet eigenvalue problem for equations with one-dimensional p-Laplacian, Abstract and Applied Analysis (2010), ID 123975, 12 pp. MR2754200

[6] C.C. Chen, C.K. Law and F.Y. Sing, Optimal lower estimates for eigenvalue ratios of Schrödinger operators and vibrating strings, Taiwanese J. Math., 9 (2005), 175-185. MR2142571(2006a:34069)

[7] A. Elbert, A half-linear second order differential equation, Colloquia Mathematica Societatis János Bolyai, 30, Qualitative Theory of Differential Equations, Szeged (Hungary) (1979), North-Holland, Amsterdam-New York, 1981, 153-180. MR680591 (84g:34008)

[8] M. Horváth, On the first two eigenvalues of Sturm-Liouville operators, Proc. Amer. Math. Soc., 131 (2003), 1215-1224. MR.1948113 (2005e:34253)

[9] M. Horváth and M. Kiss, A bound for ratios of eigenvalues of Schrödinger operators with single-well potentials, Proc. Amer. Math. Soc., 134 (2006), 1425-1434. MR2199189 (2006i:34170)

[10] M.J. Huang, On the eigenvalue ratios for vibrating strings, Proc. Amer. Math. Soc., 127 (1999), 1805-1813. MR.1621941 (99i:34119) 
[11] Y.L. Huang and C.K. Law, Eigenvalue ratios for the regular Sturm-Liouville system, Proc. Amer. Math. Soc., 124 (1996), 1427-1436. MR.1328351 (96g:34044)

[12] C.K. Law, W.C. Lian and W.C. Wang, Inverse nodal problem and Ambarzumyan problem for the p-Laplacian, Proc. Royal Soc. Edinburgh, 139A (2009), 1261-1273. MR2557322 (2011d:34022)

[13] R. Lavine, The eigenvalue gap for one-dimensional convex potentials, Proc. Amer. Math. Soc., 121 (1994), 815-821. MR.1185270 (94i:35144)

[14] W. Walter, Sturm-Liouville theory for the radial $\Delta_{p}$-operator, Math. Z., 227 (1998), 175-185. MR.1605441 (99b:35073)

Department of Applied Mathematics, National Sun Yat-Sen University, Kaohsiung 804, Taiwan, Republic of China

Department of Applied Mathematics, National Sun Yat-sen University, Kaohsiung 804, Taiwan - And - National Center for Theoretical Sciences, Taiwan, Republic of CHINA

E-mail address: law@math.nsysu.edu.tw

Department of Information Management, National Kaohsiung Marine University, Kaohsiung 811, Taiwan, Republic of China

E-mail address: wclian@mail.nkmu.edu.tw

Department of Mathematics, National Changhua University of Education, Changhua 500, Taiwan, Republic of China

E-mail address: wangwc@math.nsysu.edu.tw

E-mail address: feymann@ms39.hinet.net 